\title{
Top-quark pair production near threshold at LHC
}

\author{
Y. Kiyo ${ }^{1}$, J.H. Kühn ${ }^{1}$, S. Moch $^{2}$, M. Steinhauser ${ }^{1, a}$, P. Uwer ${ }^{3}$ \\ ${ }^{1}$ Institut für Theoretische Teilchenphysik, Universität Karlsruhe (TH), Karlsruhe Institute of Technology (KIT), 76128 Karlsruhe, Germany \\ ${ }^{2}$ Deutsches Elektronen-Synchrotron DESY, 15738 Zeuthen, Germany \\ ${ }^{3}$ Institut für Physik, Humboldt-Universität zu Berlin, 10099 Berlin, Germany
}

Received: 4 December 2008 / Revised: 7 January 2009 / Published online: 19 February 2009

(C) Springer-Verlag / Società Italiana di Fisica 2009

\begin{abstract}
The next-to-leading order analysis for the cross section for hadroproduction of top-quark pairs close to threshold is presented. Within the framework of nonrelativistic QCD a significant enhancement compared to fixed-order perturbation theory is observed which originates from the characteristic remnant of the $1 S$ peak below production threshold of top-quark pairs. The analysis includes all color-singlet and color-octet configurations of top-quark pairs in $S$-wave states and, for the dominant configurations, it employs all-order soft-gluon resummation for the hard parton cross section. Numerical results for the Large Hadron Collider at $\sqrt{s}=14 \mathrm{TeV}$ and $\sqrt{s}=10 \mathrm{TeV}$ and also for the Tevatron are presented. The possibility of a top-quark mass measurement from the invariant-mass distribution of topquark pairs is discussed.
\end{abstract}

PACS 12.38.Bx $\cdot 12.38 . \mathrm{Cy} \cdot 14.65 . \mathrm{Ha}$

\section{Introduction}

At the CERN Large Hadron Collider (LHC) the major part of top quarks are produced in pairs. Due to the experience gained at the Fermilab Tevatron [1] and the huge amount of top quarks to be produced at LHC the reconstruction of top quarks with good accuracy will be possible [2, 3]. A significant fraction of top-quark pairs will be produced close to threshold. Thus a dedicated analysis of the production cross section in this region is required which is best performed within the framework of non-relativistic QCD (NRQCD) $[4,5]$.

The production of top-antitop-quark pairs close to the kinematical threshold has received much attention in the context of precision measurement of top-quark properties at the future International Linear Collider (ILC). Theoretical calculations and dedicated experimental analyses have

a e-mail: matthias.steinhauser@uka.de demonstrated that a precise extraction of the top-quark mass, its width and the strong coupling constant is possible $[6,7]$ at the ILC. The complete next-to-next-to-leading order (NNLO) predictions have been available since many years [8]. (For earlier work see e.g [9-12].) Partial nextto-next-to-leading logarithmic (NNLL) $[13,14]$ and next-tonext-to-next-to-leading order (NNNLO) [15-17] predictions were evaluated more recently.

In contrast to the linear collider, where the physical observable is the total cross section as a function of energy, at the hadron collider one considers the invariant-mass distribution of the top-quark pairs. Since the expected uncertainty is significant larger than the one anticipated at a linear collider a next-to-leading order (NLO) analysis is probably sufficient. The calculation of the cross section within the NRQCD framework contains as building blocks the hard production cross section for a top-quark pair at threshold and the non-relativistic Green's function governing the dynamics of the would-be bound state. Both ingredients have been available in the literature since many years. In particular, the hard cross section for threshold $t \bar{t}$ production can be found in $[18,19]$. In [18] the NLO formulae were derived for quark or gluon initial states and a quarkonium in a $\mathrm{J}^{\mathrm{PC}}=0^{-+}$color-singlet state, plus possibly a parton. The general case, with the heavy quark system $(Q \bar{Q})$ in $S$-wave singlet/triplet spin state, and with color-singlet/octet configuration is given in [19], together with the corresponding results for $P$-waves. The results of $[18,19]$ were presented for stable bound states. For unstable wide resonances it is convenient to describe the bound-state dynamics through a Green's function.

Recently a calculation of top-quark threshold hadroproduction near threshold has appeared [20]. (For an early discussion along similar lines see [21].) The basic idea of our approach is similar to the one of [20]. We aim at a detailed study of the top-quark production based on NLO cross section formulae in the NRQCD framework. In our set-up all NLO sub-processes have been included, i.e., also those 
which appear for the first time in $\mathcal{O}\left(\alpha_{\mathrm{s}}^{3}\right)$. Furthermore, the matching between QCD and NRQCD as performed in [20] is slightly different from the present paper. Whereas in [20] the matching has been performed for the limit where the partonic center-of-mass energy $\hat{s}$ approaches twice the topquark mass, we include the complete dependence on $\hat{s}$ as given in $[18,19]$. Thus, formally, the result of $[20]$ is only valid for top-quark production where the velocity of both quarks is small. On the other hand, in our approach the relative velocity has to be small whereas the top-antitop-quark system can still move with high velocity. Finally, we perform a soft-gluon resummation, which enhances the cross section by a few per cent.

Our paper is organized as follows. In the next section details of the formalism used for the calculation of the NLO cross section are provided. The effects of initial-state radiation and the hard contribution are discussed in Sect. 3 and the soft-gluon resummation is performed in Sect. 4. The properties of the Green's function are summarized in Sect. 5 . In Sect. 6 the building blocks are combined and numerical results for the invariant-mass distribution are presented. Theoretical uncertainties due to scale variation and unknown higher-order corrections are estimated. A summary and conclusions are presented in Sect. 7.

\section{The production cross section}

Let us denote the (quasi-) bound state of a top and antitop quark with spin $S$ and angular momentum $L$ by $T \equiv$ ${ }^{2 S+1} L_{J}^{[1,8]}$ where the superscripts [1] and [8] denote the singlet and octet color states. The production rate is obtained from the production cross section of a top-quark pair with invariant mass $M^{2} \equiv\left(p_{t}+p_{\bar{t}}\right)^{2}$ and its evolution to a quasibound state is described by the non-relativistic QCD. The former is a hard QCD process at a distance $\sim 1 / m_{t}$ and thus computable within the conventional perturbative expansion in $\alpha_{\mathrm{s}}$.

The long-distance effects responsible for the formation of a narrow bound state are described by the squared wave function at the origin $|\Psi(0)|^{2}$ or, in the language of NRQCD, by the matrix elements

$\left\langle\left(\chi^{\dagger} \Gamma \psi\right) \cdot\left(\psi^{\dagger} \Gamma \chi\right)\right\rangle=\mathcal{N}_{\mathrm{s}} \mathcal{N}_{\mathrm{c}}|\Psi(0)|^{2}$.

Here $\mathcal{N}_{\mathrm{s}}=2 S+1$ and $\mathcal{N}_{\mathrm{c}}=1,8$ denote the number of spin and color degrees of freedom, respectively. We are interested in the differential distribution $\mathrm{d} \sigma / \mathrm{d} M$ which, for narrow resonances with mass $M_{n}$, is proportional to $\delta\left(M-M_{n}\right)$. For wide resonances, the case under consideration, it is convenient to convert the factor describing the sum over individual resonances into the non-relativistic Green's function ${ }^{1}$

$$
\begin{aligned}
& \sum_{n}\left|\Psi_{n}(0)\right|^{2} \pi \delta\left(M-M_{n}\right) \\
& \quad \rightarrow \sum_{n} \operatorname{Im} \frac{\Psi_{n}(0) \Psi_{n}^{*}(0)}{M_{n}-\left(M+\mathrm{i} \Gamma_{t}\right)}=\operatorname{Im} G\left(M+\mathrm{i} \Gamma_{t}\right),
\end{aligned}
$$

with $G\left(M+\mathrm{i} \Gamma_{t}\right) \equiv G^{[1,8]}\left(\vec{r}=0 ; M+\mathrm{i} \Gamma_{t}\right)$ being the Green's function at zero distance for the non-relativistic Schrödinger equation discussed below. Since the typical momentum scale governing the non-relativistic top-quark system $m_{t} v$ (with $m_{t} v^{2} \equiv M+\mathrm{i} \Gamma_{t}-2 m_{t}$, and $v$ being the velocity of top and antitop quarks) is in the perturbative regime, and the large top-quark width $\Gamma_{t}$ introduces an additional cutoff scale $\sqrt{m_{t} \Gamma_{t}}$, the Green's function can be evaluated perturbatively. As stated above, the present paper is concerned with the production of top-quark pairs near threshold, and thus is restricted to states with $L=0$, i.e. $T={ }^{2 S+1} S_{J}^{[1,8]}$. The contributions to the invariant-mass distribution with higher angular momentum are at least suppressed by $v^{2}$, and thus of higher order (beyond NLO).

In order to obtain experimentally measurable quantities at a hadron collider the partonic differential cross section $\mathrm{d} \hat{\sigma}_{i j \rightarrow T} / \mathrm{d} M$ is convoluted with the luminosity function,

$$
\begin{aligned}
& {\left[\frac{\mathrm{d} \mathcal{L}_{i j}}{\mathrm{~d} \tau}\right]\left(\tau, \mu_{\mathrm{f}}^{2}\right)} \\
& \quad=\int_{0}^{1} \mathrm{~d} x_{1} \int_{0}^{1} \mathrm{~d} x_{2} f_{i / P_{1}}\left(x_{1}, \mu_{\mathrm{f}}^{2}\right) f_{j / P_{2}}\left(x_{2}, \mu_{\mathrm{f}}^{2}\right) \\
& \quad \times \delta\left(\tau-x_{1} x_{2}\right),
\end{aligned}
$$

where $i, j$ refer to partons inside the hadrons $P_{1}$ and $P_{2}$ with the distribution functions $f_{i / P_{1}}$ and $f_{j / P_{2}}$. The dependence on the factorization scale $\mu_{\mathrm{f}}$ cancels in combination with the one contained in $\mathrm{d} \hat{\sigma}_{i j \rightarrow T} / \mathrm{d} M$. The differential cross section can thus be written as

$$
\begin{aligned}
& M \frac{\mathrm{d} \sigma_{P_{1} P_{2} \rightarrow T}}{\mathrm{~d} M}\left(S, M^{2}\right) \\
&=\sum_{i, j} \int_{\rho}^{1} \mathrm{~d} \tau\left[\frac{\mathrm{d} \mathcal{L}_{i j}}{\mathrm{~d} \tau}\right]\left(\tau, \mu_{\mathrm{f}}^{2}\right) M \frac{\mathrm{d} \hat{\sigma}_{i j \rightarrow T}}{\mathrm{~d} M}\left(\hat{s}, M^{2}, \mu_{\mathrm{f}}^{2}\right) .
\end{aligned}
$$

As usual $\hat{s}$ and $S$ denote the partonic and the hadronic center-of-mass energy squared, respectively, and $\tau=\hat{s} / S$. The lower limit of the $\tau$ integration is given by $\rho=M^{2} / S$. The partonic differential cross section $\mathrm{d} \hat{\sigma}_{i j \rightarrow T} / \mathrm{d} M$ consists of a factor $F$ that is evaluated in perturbative QCD, and that

\footnotetext{
${ }^{1}$ In the case of color-octet states we cannot take (2.2) literally but derive a corresponding formula within the framework of NRQCD.
} 
can be deduced from [18, 19], and a second factor, the imaginary part of the Green's function $G^{[1,8]}$,

$$
\begin{aligned}
& M \frac{\mathrm{d} \hat{\sigma}_{i j \rightarrow T}}{\mathrm{~d} M}\left(\hat{s}, M^{2}, \mu_{\mathrm{f}}^{2}\right) \\
& \quad=F_{i j \rightarrow T}\left(\hat{s}, M^{2}, \mu_{\mathrm{f}}^{2}\right) \frac{1}{m_{t}^{2}} \operatorname{Im} G^{[1,8]}\left(M+\mathrm{i} \Gamma_{t}\right),
\end{aligned}
$$

where the superscript of the Green's function refers to the color state of $T$. Equations (2.4) and (2.5) constitute our master formulae, which contain several scales and various physics contributions of different origin in factorized form. In particular, the soft dynamics of the parton distribution and real radiation is contained in the convolution of $F_{i j \rightarrow T}$ with the parton luminosity; the bound-state effects are described by $G$. Note that at NLO the Green's function $G^{[1,8]}\left(M+\mathrm{i} \Gamma_{t}\right)$ and the convolution of $F$ with the parton luminosity $(\mathcal{L} \otimes F)$ are individually independent of the renormalization scale $\mu_{\mathrm{r}}$. Thus we can discuss the two parts separately in the following two sections. Furthermore, it is simpler to assess the uncertainties for the individual contributions.

Let us at this point make a comment concerning the validity of (2.5), which makes use of the NRQCD expansion assuming $v \ll 1$, thus being limited to the threshold region. For larger invariant masses, conventional perturbation theory is applicable (see [22-24] and [25-28] for recent compilations of the total cross section and [29] for a proposal to measure the top-quark mass from the shape of $\mathrm{d} \sigma / \mathrm{d} M$ ). In the transition region the predictions from both methods are expected to coincide, as will be discussed below (cf. Fig. 6.3).

\section{Hard cross section}

In this section the ingredients for the NLO corrections to the hard cross section will be collected, which are taken from $[18,19]$. We parameterize the function $F_{i j \rightarrow T}$, representing the hard cross section for $i j \rightarrow T X$ ( $X$ stands for additional partons in the inclusive cross sections), in the following form:

$$
\begin{aligned}
F_{i j \rightarrow T}\left(\hat{s}, M^{2}, \mu_{\mathrm{f}}^{2}\right) \\
=\mathcal{N}_{i j \rightarrow T} \frac{\pi^{2} \alpha_{\mathrm{s}}^{2}\left(\mu_{\mathrm{r}}\right)}{3 \hat{s}}\left(1+\frac{\alpha_{\mathrm{s}}\left(\mu_{\mathrm{r}}\right)}{\pi} \mathcal{C}_{\mathrm{h}}\right) \\
\quad \times\left[\delta_{i j \rightarrow T} \delta(1-z)+\frac{\alpha_{\mathrm{s}}\left(\mu_{\mathrm{r}}\right)}{\pi}\left(\mathcal{A}_{\mathrm{c}}(z)+\mathcal{A}_{\mathrm{nc}}(z)\right)\right] .
\end{aligned}
$$

Here $\delta_{g g \rightarrow{ }^{1} S_{0}^{[1,8]}}=\delta_{q \bar{q} \rightarrow{ }^{3} S_{1}^{[8]}}=1$ and zero for all other $2 \rightarrow 1$ processes, and $z=M^{2} / \hat{s}$. The quantities $\mathcal{A}_{\mathrm{c}}, \mathcal{A}_{\mathrm{nc}}$, and $\mathcal{C}_{\mathrm{h}}$ all depend on $i, j$, and $T$; the functions $\mathcal{A}$ in addition depend on $z$.
The coefficients $\mathcal{C}_{\mathrm{h}}$ originate from the hard corrections to the production process. The functions $\mathcal{A}_{\mathrm{c}}$ contain the real corrections with collinear parton splitting from one of the initial partons $i, j$, and are governed by the Altarelli-Parisi splitting functions, $\mathcal{A}_{\mathrm{nc}}$ originates from non-collinear real emission. These individual contributions are manifest already in [18] and the Appendix of [19] and will be listed in the following. Note that in (3.1) we have split off the factor $\left(1+\left(\alpha_{\mathrm{s}} / \pi\right) \mathcal{C}_{\mathrm{h}}\right)$, which we attribute to hard corrections and thus treat as a multiplicative factor to the terms in square brackets.

In Table 3.1 we collect all processes of the type $i j \rightarrow T X$ at NLO which contribute in our analysis and list the corresponding normalization factors $\mathcal{N}_{i j \rightarrow T}$. Note that the production of a spin-triplet color-singlet state ${ }^{3} S_{1}^{[1]}$ via $g q$ or $q \bar{q}$ scattering is zero up to and including NLO. This is because in these channels the heavy quarks are produced through gluon splitting $g^{*} \rightarrow t \bar{t}$, which is only possible if the $t \bar{t}$ is in an octet state.

The coefficients $\mathcal{C}_{\mathrm{h}}$ are non-vanishing only for the processes which are present also in lowest order [18, 19]:

$$
\begin{aligned}
& \mathcal{C}_{\mathrm{h}} {\left[g g \rightarrow{ }^{1} S_{0}^{[1]}\right] } \\
&= \frac{\beta_{0}}{2} \ln \left(\frac{\mu_{\mathrm{r}}^{2}}{M^{2}}\right)+C_{F}\left(\frac{\pi^{2}}{4}-5\right)+C_{A}\left(1+\frac{\pi^{2}}{12}\right), \\
& \mathcal{C}_{\mathrm{h}}\left[g g \rightarrow{ }^{1} S_{0}^{[8]}\right] \\
&=\frac{\beta_{0}}{2} \ln \left(\frac{\mu_{\mathrm{r}}^{2}}{M^{2}}\right)+C_{F}\left(\frac{\pi^{2}}{4}-5\right)+C_{A}\left(3-\frac{\pi^{2}}{24}\right), \\
& \mathcal{C}_{\mathrm{h}}\left[q \bar{q} \rightarrow{ }^{3} S_{1}^{[8]}\right] \\
&=\frac{\beta_{0}}{2} \ln \left(\frac{\mu_{\mathrm{r}}^{2}}{M^{2}}\right)+C_{F}\left(\frac{\pi^{2}}{3}-8\right) \\
& \quad+C_{A}\left(\frac{59}{9}+\frac{2 \ln 2}{3}-\frac{\pi^{2}}{4}\right)-\frac{10}{9} n_{\mathrm{f}} T_{F}-\frac{16}{9} T_{F},
\end{aligned}
$$

where $\beta_{0}=(11 / 3) C_{A}-(4 / 3) n_{\mathrm{f}} T_{F}$ and $C_{F}=4 / 3, C_{A}=3$, $T_{F}=1 / 2, n_{\mathrm{f}}=5$. The last term in $\mathcal{C}_{\mathrm{h}}\left[q \bar{q} \rightarrow{ }^{3} S_{1}^{[8]}\right]$, arising from non-decoupling of the top quark in the gluon propagator, has been observed and discussed in [20]; see also [20, footnote 3, p. 73]. For the other processes the hard corrections that are of higher order; thus $\mathcal{C}_{\mathrm{h}}$ is zero at NLO:

$$
\begin{aligned}
\mathcal{C}_{\mathrm{h}}\left[g q \rightarrow{ }^{1} S_{0}^{[1,8]}\right] & =\mathcal{C}_{\mathrm{h}}\left[q \bar{q} \rightarrow{ }^{1} S_{0}^{[1,8]}\right]=\mathcal{C}_{\mathrm{h}}\left[g g \rightarrow{ }^{3} S_{1}^{[1,8]}\right] \\
& =\mathcal{C}_{\mathrm{h}}\left[g q \rightarrow{ }^{3} S_{1}^{[8]}\right]=0 .
\end{aligned}
$$

The function $\mathcal{A}_{\mathrm{c}}$ is conveniently expressed using the Altarelli-Parisi splitting functions $P_{i j}(z)$ introduced below $[18,19]$ : 
Table 3.1 Normalization factors $\mathcal{N}_{i j \rightarrow T}$ for each process for [singlet, octet] color states. $\left(N_{\mathrm{c}}=3\right.$ is used. $)$

\begin{tabular}{llllll}
\hline$g g \rightarrow{ }^{1} S_{0}^{[1,8]}$ & $g q \rightarrow{ }^{1} S_{0}^{[1,8]}$ & $q \bar{q} \rightarrow{ }^{1} S_{0}^{[1,8]}$ & $g g \rightarrow{ }^{3} S_{1}^{[1,8]}$ & $g q \rightarrow{ }^{3} S_{1}^{[1,8]}$ & $q \bar{q} \rightarrow{ }^{3} S_{1}^{[1,8]}$ \\
\hline$[1,5 / 2]$ & {$[1,5 / 2]$} & {$[3 / 4,6]$} & {$[9 / 4,18]$} & {$[0,32 / 3]$} & {$[0,32 / 3]$} \\
\hline
\end{tabular}

$$
\begin{aligned}
& \mathcal{A}_{\mathrm{c}} {\left[g g \rightarrow{ }^{1} S_{0}^{[1,8]}\right] } \\
&=(1-z) P_{g g}(z)\left\{2\left[\frac{\ln (1-z)}{1-z}\right]_{+}+\left[\frac{1}{1-z}\right]_{+} \ln \left(\frac{M^{2}}{z \mu_{\mathrm{f}}^{2}}\right)\right\} \\
& \quad-\frac{\beta_{0}}{2} \delta(1-z) \ln \left(\frac{\mu_{\mathrm{f}}^{2}}{M^{2}}\right), \\
& \mathcal{A}_{\mathrm{c}}\left[g q \rightarrow{ }^{1} S_{0}^{[1,8]}\right]=\frac{1}{2} P_{g q}(z) \ln \left(\frac{M^{2}(1-z)^{2}}{z \mu_{\mathrm{f}}^{2}}\right)+\frac{C_{F}}{2} z, \\
& \mathcal{A}_{\mathrm{c}}\left[q \bar{q} \rightarrow{ }^{1} S_{0}^{[1,8]}\right]=0, \\
& \mathcal{A}_{\mathrm{c}}\left[g g \rightarrow{ }^{3} S_{1}^{[1,8]}\right]=0, \\
& \mathcal{A}_{\mathrm{c}}\left[g q \rightarrow{ }^{3} S_{1}^{[8]}\right] \\
&=\frac{1}{2} P_{q g}(z) \ln \left(\frac{M^{2}(1-z)^{2}}{z \mu_{\mathrm{f}}^{2}}\right)+T_{F} z(1-z), \\
& \mathcal{A}_{\mathrm{c}}\left[q \bar{q} \rightarrow{ }^{3} S_{1}^{[8]}\right] \\
&=(1-z) P_{q q}(z)\left\{2\left[\frac{\ln (1-z)}{1-z}\right]_{+}+\left[\frac{1}{1-z}\right]_{+} \ln \left(\frac{M^{2}}{z \mu_{\mathrm{f}}^{2}}\right)\right\} \\
& \quad+C_{F}(1-z)-\frac{3 C_{F}}{2} \delta(1-z) \ln \left(\frac{\mu_{\mathrm{f}}^{2}}{M^{2}}\right),
\end{aligned}
$$

where the conventional plus-distribution ${ }^{2}$ was employed to regularize the singularity at $z=1$. The splitting functions $P_{i j}(z)$ are given by

$$
\begin{aligned}
& P_{g g}(z)=2 C_{A}\left[\frac{1}{1-z}+\frac{1}{z}+z(1-z)-2\right], \\
& P_{g q}(z)=C_{F}\left[\frac{1+(1-z)^{2}}{z}\right] \\
& P_{q g}(z)=T_{F}\left[z^{2}+(1-z)^{2}\right] \\
& P_{q q}(z)=2 C_{F}\left[\frac{1}{1-z}-\frac{1+z}{2}\right] .
\end{aligned}
$$

${ }^{2}$ The plus-distribution follows the prescription $\int_{0}^{1} \mathrm{~d} z\left[\frac{\ln ^{n}(1-z)}{1-z}\right]_{+} f(z) \equiv$ $\int_{0}^{1} \mathrm{~d} z \frac{\ln ^{n}(1-z)}{1-z}[f(z)-f(1)]$, where $f(z)$ is an arbitrary test function which is regular at $z=1$. It is related to the $\rho$-prescription used in [19] by $\left[\frac{\ln ^{n}(1-z)}{1-z}\right]_{+}=\left[\frac{\ln ^{n}(1-z)}{1-z}\right]_{\rho}+\frac{\ln ^{n+1}(1-\rho)}{n+1} \delta(1-z)$.
The functions $\mathcal{A}_{\text {nc }}$ are obtained from the non-collinear contributions. For spin-singlet states, we have

$$
\begin{aligned}
\mathcal{A}_{\mathrm{nc}}[ & \left.g g \rightarrow{ }^{1} S_{0}^{[1]}\right] \\
= & \frac{-C_{A}}{6 z(1-z)^{2}(1+z)^{3}}\left[12+11 z^{2}+24 z^{3}-21 z^{4}\right. \\
& -24 z^{5}+9 z^{6}-11 z^{8}+12\left(-1+5 z^{2}+2 z^{3}+z^{4}\right. \\
& \left.\left.+3 z^{6}+2 z^{7}\right) \ln z\right], \\
\mathcal{A}_{\mathrm{nc}}[ & \left.g g \rightarrow{ }^{1} S_{0}^{[8]}\right] \\
= & \frac{-C_{A}}{6 z(1-z)(1+z)^{3}}\left[12+23 z^{2}+30 z^{3}-21 z^{4}\right. \\
& \quad-24 z^{5}+9 z^{6}-6 z^{7}-23 z^{8}+\left(-12+60 z^{2}\right. \\
& \left.\left.+24 z^{3}+36 z^{4}+60 z^{6}+24 z^{7}\right) \ln z\right]\left(\frac{1}{1-z}\right), \\
\mathcal{A}_{\mathrm{nc}}[ & \left.g q \rightarrow{ }^{1} S_{0}^{[1,8]}\right]=-C_{F} \frac{1}{z}(1-z)(1-\ln z), \\
\mathcal{A}_{\mathrm{nc}}[ & \left.q \bar{q} \rightarrow{ }^{1} S_{0}^{[1]}\right]=\frac{32 C_{F}}{3 N_{\mathrm{c}}^{2}} z(1-z), \\
\mathcal{A}_{\mathrm{nc}}[ & \left.q \bar{q} \rightarrow{ }^{1} S_{0}^{[8]}\right]=\frac{32 B_{F}}{3 N_{\mathrm{c}}^{2}} z(1-z),
\end{aligned}
$$

where $B_{F}=\left(N_{\mathrm{c}}^{2}-4\right) /\left(4 N_{\mathrm{c}}\right)$ with $N_{\mathrm{c}}=3$. Note that $\mathcal{A}_{\mathrm{nc}}\left[\mathrm{g} g \rightarrow{ }^{1} S_{0}^{[8]}\right]$ is singular at $z=1$, and regularized by the plus-prescription. For spin-triplet states, one obtains

$$
\begin{aligned}
\mathcal{A}_{\mathrm{nc}}[ & \left.g g \rightarrow{ }^{3} S_{1}^{[1]}\right] \\
= & \frac{256 B_{F}}{6 C_{F} N_{\mathrm{c}}^{2}} \frac{z}{(1-z)^{2}(1+z)^{3}} \\
& \times\left[2+z+2 z^{2}-4 z^{4}-z^{5}+2 z^{2}\left(5+2 z+z^{2}\right) \ln z\right], \\
\mathcal{A}_{\mathrm{nc}}[ & \left.g g \rightarrow{ }^{3} S_{1}^{[8]}\right] \\
= & \frac{1}{36 z(1-z)^{2}(1+z)^{3}}\left[108+153 z+400 z^{2}+65 z^{3}\right. \\
& -356 z^{4}-189 z^{5}-152 z^{6}-29 z^{7}+(108 z \\
& \left.\left.+756 z^{2}+432 z^{3}+704 z^{4}+260 z^{5}+76 z^{6}\right) \ln z\right] \\
\mathcal{A}_{\mathrm{nc}}[ & \left.g q \rightarrow{ }^{3} S_{1}^{[8]}\right] \\
= & \frac{T_{F}}{4}(1-z)(1+3 z)+\frac{C_{A}}{4 C_{F}} \frac{1}{z}\left[(1-z)\left(2+z+2 z^{2}\right)\right. \\
& +2 z(1+z) \ln z], \\
\mathcal{A}_{\mathrm{nc}}[ & {\left[q \bar{q} \rightarrow{ }^{3} S_{1}^{[8]}\right] } \\
= & -\left[C_{F}(1-z)^{2}+\frac{C_{A}}{3}\left(1+z+z^{2}\right)\right]\left(\frac{1}{1-z}\right)_{+}
\end{aligned}
$$


Table 3.2 The convolution $\mathcal{L} \otimes F$ for $\mathrm{LHC}$ at the reference point $M=2 m_{t}$, for the production of color singlet and octet states. The three columns correspond to the scale choices $\mu_{\mathrm{r}}=\mu_{\mathrm{f}}=\left(m_{t}, 2 m_{t}, 4 m_{t}\right)$

\begin{tabular}{lcccccc}
\hline & $\mathcal{L} \otimes F\left[i j \rightarrow T^{[1]}\right] \times 10^{6}\left[\mathrm{GeV}^{-2}\right]$ & \multicolumn{2}{c}{$\mathcal{L} \otimes F\left[i j \rightarrow T^{[8]}\right] \times 10^{6}\left[\mathrm{GeV}^{-2}\right]$} \\
\hline$g g \rightarrow{ }^{1} S_{0}^{[1,8]}$ & 20.7 & 21.2 & 20.9 & 63.2 & 62.7 & 60.2 \\
$g q \rightarrow{ }^{1} S_{0}^{[1,8]}$ & -0.795 & -1.74 & -2.19 & -1.99 & -4.36 & -5.47 \\
$q \bar{q} \rightarrow{ }^{1} S_{0}^{[1,8]}$ & 0.00664 & 0.00509 & 0.00398 & 0.0166 & 0.0127 & 0.00995 \\
$g g \rightarrow{ }^{3} S_{1}^{[1,8]}$ & 0.175 & 0.127 & 0.0936 & 6.06 & 4.26 & 3.07 \\
$g q \rightarrow{ }^{3} S_{1}^{[8]}$ & - & - & - & 3.99 & 1.68 & 0.279 \\
$q \bar{q} \rightarrow{ }^{3} S_{1}^{[8]}$ & - & - & - & 23.1 & 23.8 & 23.6 \\
Total: $\left({ }^{1} S_{0}+{ }^{3} S_{1}\right)^{[1,8]}$ & 20.0 & 19.6 & 18.8 & 94.3 & 88.1 & 81.8 \\
\hline
\end{tabular}

The function $\mathcal{A}_{\text {nc }}\left[q \bar{q} \rightarrow{ }^{3} S_{1}^{[8]}\right]$ is also defined with the plusprescription. The leading singular behavior of $\mathcal{A}_{\mathrm{nc}}$ is given by $\mathcal{A}_{\mathrm{nc}}(z) \stackrel{z \rightarrow 1}{\sim}-C_{A} /(1-z)_{+}$both for $g g \rightarrow{ }^{1} S_{0}^{[8]}$ and $q \bar{q} \rightarrow{ }^{3} S_{1}^{[8]}$. In the soft limit its behavior is insensitive to the details of the bound state and only depends on its color configuration.

It is instructive to discuss the relation between the normalizations of different processes leading to the same bound state. For instance, the normalization $\mathcal{N}_{i j \rightarrow T}$ (see Table 3.1) for the process $g q \rightarrow{ }^{1} S_{0}^{[1,8]} X$ is fixed by $g g \rightarrow{ }^{1} S_{0}^{[1,8]}$, because in the collinear limit this cross section factorizes into the corresponding LO process and the $P_{g q}$ splitting function. As a consequence, the cancellation of the factorization scale dependence happens among the $g g$ and $g q$ initiated reactions. Similarly, the normalization of $g q \rightarrow{ }^{3} S_{1}^{[8]}$ is fixed by $q \bar{q} \rightarrow{ }^{3} S_{1}^{[8]}$. In contrast, the processes $q \bar{q} \rightarrow{ }^{1} S_{0}^{[1,8]}$ and $g g \rightarrow{ }^{3} S_{1}^{[1,8]}$ are forbidden at LO; hence the corrections have to be collinearly finite. In comparison to [20] the combinations $\mathcal{A}_{\mathrm{c}}+\mathcal{A}_{\mathrm{nc}}$ include terms that vanish in the limit $z \rightarrow 1$. Furthermore, subprocesses that appear for the first time in $\mathcal{O}\left(\alpha_{\mathrm{s}}^{3}\right)$ were neglected in [20]. The relative size of these terms will be addressed below.

Let us now start the numerical analysis. The partonic cross sections have to be convoluted with the partondistribution functions (PDFs) in order to arrive at the hadronic cross section. We use the CTEQ6.5 [30] set for the PDFs and take $\alpha_{\mathrm{S}}^{(5)}\left(M_{Z}\right)=0.118, m_{t}=172.4 \mathrm{GeV}$ and $\sqrt{S}=14 \mathrm{TeV}$ as input values. The running of $\alpha_{\mathrm{s}}^{(5)}\left(\mu_{\mathrm{r}}\right)$, which is the input for the partonic cross sections, is evaluated with the help of RunDec [31], using the four-loop approximation of the $\beta$ function. This leads to $\alpha_{\mathrm{s}}^{(5)}\left(\mu_{\mathrm{r}}\right)=$ $(0.1077,0.09832,0.09050)$ for $\mu_{\mathrm{r}}=\left(m_{t}, 2 m_{t}, 4 m_{t}\right)$. Furthermore, we identify renormalization and factorization scales $\left(\mu_{\mathrm{f}}=\mu_{\mathrm{r}}\right)$.

As stated above, the cross section factors into the convolution $\mathcal{L} \otimes F$ and the Green's function. To discuss the relative importance of the various contributions individually, the results for the subprocesses without the factor $\operatorname{Im} G\left(M+\mathrm{i} \Gamma_{t}\right) / m_{t}^{2}$ are given in Table 3.2. Note that colorsinglet $t \bar{t}$ production is dominated by $g g \rightarrow{ }^{1} S_{0}^{[1]}$. Color- octet production is dominated by $g g \rightarrow{ }^{1} S_{0}^{[8]}$ plus a $25 \%$ contribution from $q \bar{q} \rightarrow{ }^{3} S_{1}^{[8]}$. The size of the remaining subprocesses (neglected in [20]) amounts to five to ten percent and is strongly scale dependent. The variation of $\mu$ (recall $\mu=\mu_{\mathrm{f}}=\mu_{\mathrm{r}}$ ) between $m_{t}$ and $4 m_{t}$ leads to changes of $\mathcal{L} \otimes F$ by $\pm 3 \%$ and $\pm 7 \%$ for the total singlet and octet production, respectively. In these channels the real radiation of partons contains large logarithmic contributions in the NLO corrections. In combination with the rapidly varying parton luminosity these logarithms make up for a major part of the numbers quoted in Table 3.2. The origin of these large logarithms can be traced to the singular behavior of the cross section near $z \approx 1$, regularized by plus-distributions. There exists well established technology for the resummation of these large logarithms to all orders in perturbation theory. We will address this issue next.

\section{Soft-gluon resummation}

The parton channels which exhibit enhancement due to softgluon emission are $g g \rightarrow{ }^{1} S_{0}^{[1]}, g g \rightarrow{ }^{1} S_{0}^{[8]}$, and $q \bar{q} \rightarrow$ ${ }^{3} S_{1}^{[8]}$ (see (3.4) and (3.7)). The relevant logarithms are contained both in $\mathcal{A}_{\mathrm{c}}$ (from initial-state radiation) and $\mathcal{A}_{\mathrm{nc}}$ (from FSR) and read for the three leading processes

$$
\begin{aligned}
& \mathcal{A}_{\text {thrlog }}\left[g g \rightarrow{ }^{1} S_{0}^{[1]}\right] \\
& =4 C_{A} D_{1}-2 C_{A} \ln \left(\frac{\mu_{\mathrm{f}}^{2}}{M^{2}}\right) D_{0}-\frac{\beta_{0}}{2} \delta(1-z) \ln \left(\frac{\mu_{\mathrm{f}}^{2}}{M^{2}}\right), \\
& \mathcal{A}_{\text {thrlog }}\left[g g \rightarrow{ }^{1} S_{0}^{[8]}\right] \\
& =\mathcal{A}_{\text {thrlog }}\left[g g \rightarrow{ }^{1} S_{0}^{[1]}\right]-C_{A} D_{0}, \\
& \mathcal{A}_{\text {thrlog }}\left[q \bar{q} \rightarrow{ }^{3} S_{1}^{[8]}\right] \\
& =4 C_{F} D_{1}-\left(2 C_{F} \ln \left(\frac{\mu_{\mathrm{f}}^{2}}{M^{2}}\right)+C_{A}\right) D_{0} \\
& \quad-\frac{3 C_{F}}{2} \delta(1-z) \ln \left(\frac{\mu_{\mathrm{f}}^{2}}{M^{2}}\right)
\end{aligned}
$$

where $D_{l}=\left[\ln ^{l}(1-z) /(1-z)\right]_{+}$denote the plus-distributions and all $\ln \mu_{\mathrm{f}}^{2} / M^{2}$ parts are included in the definition of 
the threshold logarithm. Whether the threshold logarithms are enhanced or not depends on the behavior of the parton luminosity functions near the kinematical end point $\tau=\rho$. To investigate the size of the threshold logarithms, we evaluate the contribution of the factorized hard-scattering contribution convoluted with the PDFs, i.e. $\mathcal{L} \otimes F$ separately for the three contributions of tree-level, singular and regular terms. (The hard corrections $\left(1+\left(\alpha_{\mathrm{s}} / \pi\right) \mathcal{C}\right)$ are common to all.) The threshold-enhanced contributions are defined in (4.1) and correspond exactly to the terms included in [20], while regular terms correspond to the remainder of $\mathcal{A}_{\mathrm{c}}+\mathcal{A}_{\mathrm{nc}}$ in (3.4) and (3.7) without plus-distributions. For $M=2 m_{t}$ and $\sqrt{S}=14 \mathrm{TeV}$ we obtain the following results:

$$
\begin{aligned}
& (\mathcal{L} \otimes F)\left[g g \rightarrow{ }^{1} S_{0}^{[1]}\right] \\
& \quad=\left\{\begin{array}{l}
14.5+(4.53+1.68)_{\mathcal{A}} \\
14.0+(5.66+1.58)_{\mathcal{A}} \\
13.0+(6.37+1.48)_{\mathcal{A}}
\end{array}\right\} \times 10^{-6} \mathrm{GeV}^{-2}, \\
& (\mathcal{L} \otimes F)\left[g g \rightarrow{ }^{1} S_{0}^{[8]}\right] \\
& \quad=\left\{\begin{array}{l}
39.3+(16.6+7.26)_{\mathcal{A}} \\
37.4+(18.8+6.52)_{\mathcal{A}} \\
34.4+(20.0+5.83)_{\mathcal{A}}
\end{array}\right\} \times 10^{-6} \mathrm{GeV}^{-2}, \\
& (\mathcal{L} \otimes F)\left[q \bar{q} \rightarrow{ }^{3} S_{1}^{[8]}\right] \\
& \quad=\left\{\begin{array}{l}
16.7+(3.50+2.91)_{\mathcal{A}} \\
16.8+(3.41+3.56)_{\mathcal{A}} \\
16.4+(3.28+3.97)_{\mathcal{A}}
\end{array}\right\} \times 10^{-6} \mathrm{GeV}^{-2} .
\end{aligned}
$$

The three lines correspond to $\mu=\mu_{\mathrm{f}}=\mu_{\mathrm{r}}=$ $\left(m_{t}, 2 m_{t}, 4 m_{t}\right)$. We note that in all three cases the contribution of the threshold-enhanced terms from (4.1) is large, although the regular terms in the case of $q \bar{q} \rightarrow{ }^{3} S_{1}^{[8]}$ are of the same order. Technically the matching applied in [20] corresponds to neglecting all terms which vanish exactly at threshold, that is, for $z=1$, i.e. (3.6) and (3.7) of Sect. 3. The regular terms in (4.2), which have not been accounted for in the recent analysis of [20], are of the same order as the NLO sub-processes as given in Table 3.2.

Threshold resummation proceeds conveniently in Mellin space. To that end we calculate the Mellin moments with respect to $z=M^{2} / \hat{s}$ according to

$$
F_{i j \rightarrow T}^{N}\left(M^{2}, \mu_{\mathrm{f}}^{2}\right)=\int_{0}^{1} \mathrm{~d} z z^{N-1} F_{i j \rightarrow T}\left(\hat{s}, M^{2}, \mu_{\mathrm{f}}^{2}\right) .
$$

Then, the Mellin-space expression for the thresholdenhanced terms listed in (4.1) read (see also [32, 33])

$$
\begin{aligned}
& \mathcal{A}_{\mathrm{thrlog}}^{N}\left[g g \rightarrow{ }^{1} S_{0}^{[1]}\right] \\
& =2 C_{A} \ln ^{2} N+C_{A} \ln N\left(4 \gamma_{\mathrm{E}}-2 \ln \left(\frac{M^{2}}{\mu_{\mathrm{f}}^{2}}\right)\right) \\
& \quad+C_{A}\left(2 \zeta_{2}+2 \gamma_{\mathrm{E}}^{2}-2 \gamma_{\mathrm{E}} \ln \left(\frac{M^{2}}{\mu_{\mathrm{f}}^{2}}\right)\right)+\frac{1}{2} \beta_{0} \ln \left(\frac{M^{2}}{\mu_{\mathrm{f}}^{2}}\right), \\
& \mathcal{A}_{\mathrm{thrlog}}^{N}\left[g g \rightarrow{ }^{1} S_{0}^{[8]}\right] \\
& =\mathcal{A}_{\mathrm{thrlog}}^{N}\left[g g \rightarrow{ }^{1} S_{0}^{[8]}\right]+C_{A} \ln N+C_{A} \gamma_{\mathrm{E}}, \\
& \mathcal{A}_{\mathrm{thrlog}}^{N}\left[q \bar{q} \rightarrow{ }^{3} S_{1}^{[8]}\right] \\
& =2 C_{F} \ln { }^{2} N+C_{F} \ln N\left(4 \gamma_{\mathrm{E}}-2 \ln \left(\frac{M^{2}}{\mu_{\mathrm{f}}^{2}}\right)\right)+C_{A} \ln N \\
& \quad+C_{F}\left(2 \zeta_{2}+2 \gamma_{\mathrm{E}}^{2}+\frac{3}{2} \ln \left(\frac{M^{2}}{\mu_{\mathrm{f}}^{2}}\right)-2 \gamma_{\mathrm{E}} \ln \left(\frac{M^{2}}{\mu_{\mathrm{f}}^{2}}\right)\right) \\
& \quad+C_{A} \gamma_{\mathrm{E}},
\end{aligned}
$$

where we have kept all dominant terms in the large- $N$ limit and neglected power-suppressed terms of order $1 / N . \gamma_{\mathrm{E}}$ is the Euler-Mascheroni constant $\left(\gamma_{\mathrm{E}}=0.577215 \ldots\right)$.

The resummed expressions (defined in the $\overline{\mathrm{MS}}$-scheme) for the individual color structures of the hard cross sections $F$ of (3.1) are given by a single exponential in Mellin space (see e.g. [33-35])

$$
\begin{aligned}
\frac{F_{i j \rightarrow T}^{N}\left(M^{2}, \mu_{\mathrm{f}}^{2}\right)}{F_{i j \rightarrow T}^{(0), N}\left(M^{2}, \mu_{\mathrm{f}}^{2}\right)}= & g_{i j \rightarrow T}^{0}\left(m_{t}^{2}, \mu_{\mathrm{f}}^{2}, \mu_{\mathrm{r}}^{2}\right) \Delta_{i j \rightarrow T}^{N+1}\left(m_{t}^{2}, \mu_{\mathrm{f}}^{2}, \mu_{\mathrm{r}}^{2}\right) \\
& +\mathcal{O}\left(N^{-1} \ln ^{n} N\right),
\end{aligned}
$$

where $F_{i j \rightarrow T}^{(0), N}$ denotes the tree-level term in (3.1) and the exponents are commonly expressed as

$\ln \Delta_{i j \rightarrow T}^{N}=\ln N \cdot g_{i j}^{1}(\lambda)+g_{i j \rightarrow T}^{2}(\lambda)+\cdots$,

where $\lambda=\beta_{0} \alpha_{\mathrm{s}} \ln N /(4 \pi)$. To next-to-leading logarithmic (NLL) accuracy the (universal) functions $g_{i j}^{1}$ as well as the functions $g_{i j \rightarrow T}^{2}$ are relevant in (4.6); see [25] for the extension to NNLL accuracy. Explicit expressions are

$$
\begin{aligned}
& g_{q \bar{q}}^{1}= \frac{A_{q}^{(1)}}{\beta_{0}}\left[2-2 \ln (1-2 \lambda)+\lambda^{-1} \ln (1-2 \lambda)\right], \\
& g_{q \bar{q} \rightarrow T[1]}^{2} \rightarrow\left(\frac{A_{q}^{(1)} \beta_{1}}{\beta_{0}^{3}}-\frac{A_{q}^{(2)}}{\beta_{0}^{2}}\right)[2 \lambda+\ln (1-2 \lambda)] \\
&+\frac{A_{q}^{(1)} \beta_{1}}{2 \beta_{0}^{3}} \ln ^{2}(1-2 \lambda)-2 \frac{A_{q}^{(1)}}{\beta_{0}} \gamma_{\mathrm{E}} \ln (1-2 \lambda) \\
&+\ln \left(\frac{M^{2}}{\mu_{\mathrm{r}}^{2}}\right) \frac{A_{q}^{(1)}}{\beta_{0}} \ln (1-2 \lambda)+2 \ln \left(\frac{\mu_{\mathrm{f}}^{2}}{\mu_{\mathrm{r}}^{2}}\right) \frac{A_{q}^{(1)}}{\beta_{0}} \lambda, \\
& g_{q \bar{q} \rightarrow T[8]}^{2}=g_{q \bar{q} \rightarrow T[1]}^{2}-\frac{D_{Q \bar{Q}}^{(1)}}{2 \beta_{0}} \ln (1-2 \lambda),
\end{aligned}
$$


where the full dependence on $\mu_{\mathrm{r}}$ and $\mu_{\mathrm{f}}$ has been kept. The gluonic expressions $g_{g g}^{1}$ and $g_{g g \rightarrow T}^{2}$ are obtained with the obvious replacement $A_{q}^{(i)} \rightarrow A_{g}^{(i)}$. The perturbative expansions of the anomalous dimensions are universal and well known. We have [36]

$A_{q}^{(1)}=4 C_{F}$,

$A_{q}^{(2)}=8 C_{F}\left[\left(\frac{67}{18}-\zeta_{2}\right) C_{A}-\frac{5}{9} n_{\mathrm{f}}\right]$,

$D_{Q \bar{Q}}^{(1)}=4 C_{A}$,

and all gluonic quantities are given by multiplying $A_{q}^{(i)}$ by $C_{A} / C_{F}$. We also give explicit results for the matching functions $g_{i j \rightarrow T}^{0}$ in (4.5),

$$
\begin{aligned}
& g_{g g \rightarrow T[1]}^{0}=1+\frac{\alpha_{\mathrm{s}}}{\pi}\left\{C_{A}\left[2 \zeta_{2}+2 \gamma_{\mathrm{E}}^{2}-2 \gamma_{\mathrm{E}} \ln \left(\frac{M^{2}}{\mu_{\mathrm{f}}^{2}}\right)\right]\right. \\
&\left.+\frac{1}{2} \beta_{0} \ln \left(\frac{M^{2}}{\mu_{\mathrm{f}}^{2}}\right)\right\}, \\
& g_{g g \rightarrow T[8]}^{0}= g_{g g \rightarrow T[1]}^{0}+\frac{\alpha_{\mathrm{s}}}{\pi} C_{A} \gamma_{\mathrm{E}}, \\
& g_{q \bar{q} \rightarrow T[8]}^{0}=1+\frac{\alpha_{\mathrm{s}}}{\pi}\left\{C _ { F } \left[2 \zeta_{2}+2 \gamma_{\mathrm{E}}^{2}+\frac{3}{2} \ln \left(\frac{M^{2}}{\mu_{\mathrm{f}}^{2}}\right)\right.\right. \\
& \\
&\left.\left.-2 \gamma_{\mathrm{E}} \ln \left(\frac{M^{2}}{\mu_{\mathrm{f}}^{2}}\right)\right]+C_{A} \gamma_{\mathrm{E}}\right\} .
\end{aligned}
$$

For phenomenological applications [37, 38] of soft-gluon resummation at the parton level one introduces an improved (resummed) hard cross section $F^{\text {res }}$, which is obtained by an inverse Mellin transformation, as follows:

$$
\begin{aligned}
& F_{i j \rightarrow T}^{\mathrm{res}}\left(\hat{s}, M^{2}, \mu_{\mathrm{f}}^{2}\right) \\
& =\int_{c-\mathrm{i} \infty}^{c+\mathrm{i} \infty} \frac{\mathrm{d} N}{2 \pi \mathrm{i}} x^{-N}\left(F_{i j \rightarrow T}^{N}\left(M^{2}, \mu_{\mathrm{f}}^{2}\right)\right. \\
& \left.\quad-\left.F_{i j \rightarrow T}^{N}\left(M^{2}, \mu_{\mathrm{f}}^{2}\right)\right|_{\mathrm{NLO}}\right)+F_{i j \rightarrow T}^{\mathrm{NLO}}\left(\hat{s}, M^{2}, \mu_{\mathrm{f}}^{2}\right) .
\end{aligned}
$$

Here $F_{i j \rightarrow T}^{\mathrm{NLO}}$ is the standard fixed-order cross section at NLO in QCD, while $\left.F_{i j \rightarrow T}^{N}\right|_{\mathrm{NLO}}$ is the perturbative truncation at the same order in $\alpha_{\mathrm{s}}$ obtained by employing (4.4). This is to say that for the matching we have fully expanded all formulae consistently to $\mathcal{O}\left(\alpha_{\mathrm{s}}\right)$. This adds the hard coefficients $\mathcal{C}_{\mathrm{h}}$ of (3.2) to the results (4.4) and (4.9). In this way, the righthand side of (4.10) reproduces the fixed-order results and resums soft-gluon effects beyond NLO to NLL accuracy.

In Sect. 6 we employ (4.10) for phenomenological predictions by performing the inverse Mellin transform numerically. To that end, one should note that the treatment of the
Table 4.1 Comparison of the NLO and resummed result of the convolution $\mathcal{L} \otimes F$ (in $10^{-6} \mathrm{GeV}^{-2}$ ) for LHC at the reference point $M=2 m_{t}$. The three columns correspond to the scale choices $\mu_{\mathrm{r}}=$ $\mu_{\mathrm{f}}=\left(m_{t}, 2 m_{t}, 4 m_{t}\right)$. The NLO results can also be found in Table 3.2

\begin{tabular}{lllllll}
\hline & NLO & \multicolumn{5}{c}{ Resummed } \\
\hline$g g \rightarrow{ }^{1} S_{0}^{[1]}$ & 20.7 & 21.2 & 20.9 & 22.0 & 23.2 & 24.0 \\
$g g \rightarrow{ }^{1} S_{0}^{[8]}$ & 63.2 & 62.7 & 60.2 & 67.8 & 69.7 & 70.6 \\
$q \bar{q} \rightarrow{ }^{3} S_{1}^{[8]}$ & 23.1 & 23.8 & 23.6 & 23.8 & 24.0 & 23.6 \\
\hline
\end{tabular}

precise numerical matching to the exact NLO hard cross section is a matter of choice since different schemes lead only to differences which are formally of higher order. We have found that the application of the resummed result is well justified when the kinetic energy of the top-quark pair is a few $\mathrm{GeV}$ or less, see e.g. [25], where the precise numerical value is not important. Another issue concerns the constant terms in (4.9), which are sometimes modified to include formally sub-leading (but numerically not insignificant) terms; see for instance $[37,38]$. As just explained, in the present analysis we adopt the minimal approach, i.e. we apply (4.9) (including the hard coefficients $\mathcal{C}_{\mathrm{h}}$ of (3.2)) and account for all regular terms in (4.2) through matching to NLO.

In Table 4.1 we compare the fixed-order NLOand resummed result of the convolution $\mathcal{L} \otimes F$. One observes an enhancement up to about $10 \%$ depending on the process.

\section{Bound-state corrections}

Let us next discuss the bound-state corrections. As mentioned above, the convolution of $F_{i j \rightarrow T}$ with the parton luminosities provides the normalization of the differential cross section, while its shape is mainly determined by the non-relativistic Green's function. The latter describes the long-distance evolution of the top-quark pair produced near threshold. The kinematics of the produced top-quark pair is non-relativistic, and the dynamics is governed by exchange of potential gluons leading to the formation of quasibound states. The corresponding potential is given at NLO by

$$
\begin{aligned}
\widetilde{V}_{C}^{[1,8]}(\vec{q})= & -\frac{4 \pi \alpha_{\mathrm{s}}\left(\mu_{\mathrm{r}}\right) C^{[1,8]}}{\vec{q}^{2}} \\
& \times\left[1+\frac{\alpha_{\mathrm{s}}\left(\mu_{\mathrm{r}}\right)}{4 \pi}\left(\beta_{0} \ln \frac{\mu_{\mathrm{r}}^{2}}{\vec{q}^{2}}+a_{1}\right)\right],
\end{aligned}
$$

with $C^{[1]}=C_{F}=4 / 3$ and $C^{[8]}=C_{F}-C_{A} / 2=-1 / 6$, and $a_{1}=(31 / 9) C_{A}-(20 / 9) T_{F} n_{\mathrm{f}}$. 
The color-singlet Green's function feels an attractive force, the color-octet Green's function is governed by repulsion and thus does not develop a bound state. They are both defined as the solutions of the Schrödinger equations

$$
\begin{aligned}
& \left\{2 m_{t}+\left[\frac{(-\mathrm{i} \nabla)^{2}}{m_{t}}+V_{C}^{[1,8]}(\vec{r})\right]-\left(M+\mathrm{i} \Gamma_{t}\right)\right\} \\
& \times G^{[1,8]}\left(\vec{r} ; M+\mathrm{i} \Gamma_{t}\right)=\delta^{(3)}(\vec{r}) .
\end{aligned}
$$

For the Green's function at zero distance, the NLO result is known in a compact form [39] (see also [14])

$$
\begin{aligned}
G^{[1,8]}\left(M+\mathrm{i} \Gamma_{t}\right) & G^{[1,8]}\left(\vec{r}=0 ; M+\mathrm{i} \Gamma_{t}\right) \\
= & \frac{C^{[1,8]} \alpha_{\mathrm{s}}\left(\mu_{\mathrm{r}}\right) m_{t}^{2}}{4 \pi}\left[g_{\mathrm{LO}}+\frac{\alpha_{\mathrm{s}}\left(\mu_{\mathrm{r}}\right)}{4 \pi} g_{\mathrm{NLO}}+\cdots\right], \\
g_{\mathrm{LO}}=- & \frac{1}{2 \kappa}+L-\psi^{(0)}, \\
g_{\mathrm{NLO}}= & \beta_{0}\left[L^{2}-2 L\left(\psi^{(0)}-\kappa \psi^{(1)}\right)+\kappa \psi^{(2)}\right. \\
& +\left(\psi^{(0)}\right)^{2}-3 \psi^{(1)}-2 \kappa \psi^{(0)} \psi^{(1)} \\
& \left.+4 F_{3}(1,1,1,1 ; 2,2,1-\kappa ; 1)\right] \\
& +a_{1}\left[L-\psi^{(0)}+\kappa \psi^{(1)}\right],
\end{aligned}
$$

with

$\kappa \equiv \frac{\mathrm{i} C^{[1,8]} \alpha_{\mathrm{s}}\left(\mu_{\mathrm{r}}\right)}{2 v}, \quad v=\sqrt{\frac{M+\mathrm{i} \Gamma_{t}-2 m_{t}}{m_{t}}}$.

Here $L=\ln \left(\mathrm{i} \mu_{\mathrm{r}} /\left(2 m_{t} v\right)\right)$ and $\psi^{(n)}=\psi^{(n)}(1-\kappa)$ is the $n$th derivative of $\psi(z) \equiv \gamma_{\mathrm{E}}+(\mathrm{d} / \mathrm{d} z) \ln \Gamma(z)$ with argument $(1-\kappa)$. The Green's function in (5.3) correctly reproduces all the NLO terms in NRQCD; however, it is not sufficient to describe the behavior of the Green's function in the vicinity of bound-state poles. It is because the exact solution to the Schrödinger equation has only single poles in the bound-state energy $G^{[1]} \sim|\Psi(0)|^{2} /\left(M_{n}-M-\right.$ $\mathrm{i} \Gamma_{t}$ ), while (5.3) is an expansion around the LO boundstate poles and thus has multiple poles of the form $G \sim$ $\left|\Psi_{n}^{(0)}(0)\right|^{2} /\left(M_{n}^{(0)}-M\right)^{k}(k=1,2$ at the NLO). However, resummation of these multiple poles into single poles is straightforward and well known. We refer to [39] for further details.

In Fig. 5.1 we show the imaginary parts of the colorsinglet and color-octet Green's functions in the threshold region. As input we use $m_{t}^{\mathrm{PS}}=170.1 \mathrm{GeV}$, which to NLO accuracy corresponds to $m_{t}=172.4 \mathrm{GeV}$ [1], and $\Gamma_{t}=1.36 \mathrm{GeV}$ [40-42]. At NLO the Green's function is separately renormalization-scale invariant and we are free to chose $\mu_{\mathrm{r}}$ independent from the hard process. A wellmotivated physical scale is $\mu_{\mathrm{s}}=m_{t} C_{F} \alpha_{\mathrm{s}}\left(\mu_{\mathrm{s}}\right)=32.21 \mathrm{GeV}$, which corresponds to twice the inverse Bohr radius. The corresponding $\alpha_{\mathrm{s}}$ value used in Fig. 5.1 is $\alpha_{\mathrm{s}}^{\left(n_{\mathrm{f}}=5\right)}\left(\mu_{\mathrm{s}}\right)=$ 0.1401. It has been observed that the color-singlet Coulomb Green's function has a well-convergent perturbative series for this choice of scale [43].

In order to see the effect of Coulomb resummation, we plot for both color states three lines: the full Green's function (solid line) and the expansion of $G$ in fixed order up to $\mathcal{O}\left(\alpha_{\mathrm{s}}\right)$ with and without top-quark width (dashed/dotted). The upper three lines in Fig. 5.1 correspond to the colorsinglet case and the lower three to the color-octet one. The color-singlet Green's function shows a pronounced peak which corresponds to the $t \bar{t}$ resonance below $2 m_{t}$, while for the color octet there is no enhancement. Note that the curve for the full octet Green's function is very close to the one-loop expansion (taking into account the finite top-quark width). Thus for the color-octet state the Coulomb resummation effect is negligible. In addition, one more line (dashdotted) for the color-singlet Green's function is plotted including the NNLO Coulomb potential, which is useful to estimate yet unknown bound-state corrections to the NLO color-singlet Green's function. As input value we again adopt the PS top-quark mass [44] given above. Note that in the absence of a full NNLO result for the Green's function and hard correction, this improved Green's function would not be sufficient for a full NNLO prediction. Nevertheless, the difference between solid and dash-dotted curves gives an

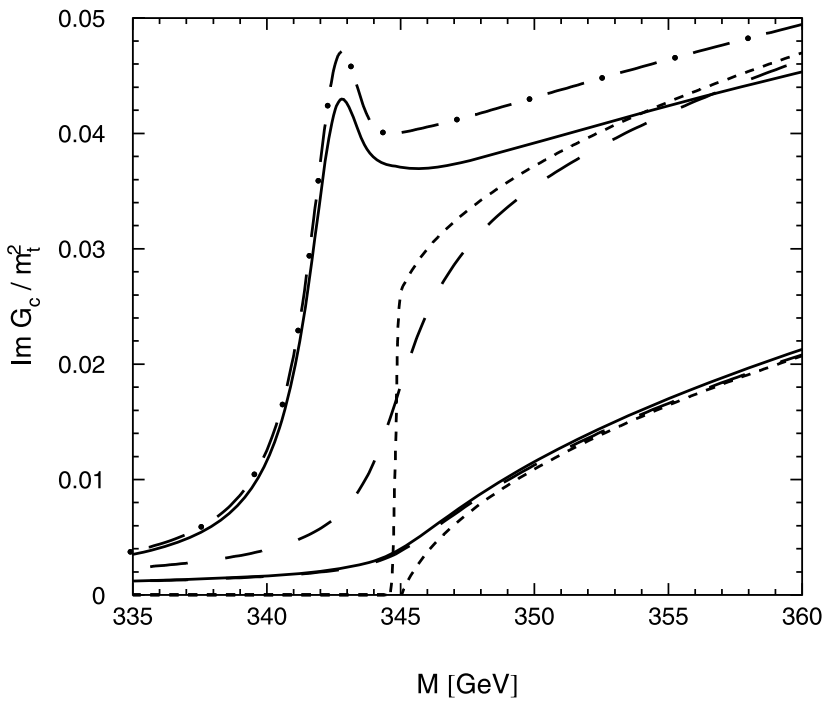

Fig. 5.1 Imaginary part of the Green's functions for the color-singlet (upper solid line) and color-octet (lower solid line) cases as functions of the top-quark invariant mass. For comparison, also the expansions of $G$ in fixed order up to $\mathcal{O}\left(\alpha_{\mathrm{s}}\right)$ with (dashed) and without (dotted line) $\Gamma_{t}$ are plotted. The imaginary part of the NNLO Green's function for the color-singlet case is shown as dash-dotted line 
indication of the intrinsic uncertainties of the Green's function, which is roughly $10 \%$.

The expansion of $G$ up to $\mathcal{O}\left(\alpha_{\mathrm{s}}\right)$ is obtained from the $g_{\mathrm{LO}}$ in (5.3) as

$$
\begin{aligned}
\frac{1}{m_{t}^{2}} \operatorname{Im} G_{\mathrm{c}}= & \operatorname{Im}\left[\frac{v}{4 \pi}\left(\mathrm{i}+\frac{\alpha_{\mathrm{s}} C^{[1,8]}}{v}\left[\frac{\mathrm{i} \pi}{2}-\ln v\right]\right)\right] \\
& +\mathcal{O}\left(\alpha_{\mathrm{s}}^{2}\right)
\end{aligned}
$$

In the zero-width limit ( $\mathrm{i} \Gamma_{t} \rightarrow+\mathrm{i} 0$ ), the color-singlet curve for the expansion exhibits a step of height $\alpha_{\mathrm{S}} C_{F} / 8$ (for $M \rightarrow 2 m_{t}$ ), and the color-octet curve formally becomes negative for $v \leq-\alpha_{\mathrm{S}} C^{[8]} \pi / 2$ which corresponds to $M-$ $2 m_{t}<0.23 \mathrm{GeV}$. Both for the singlet and octet case, the fixed-order result without $\Gamma_{t}$, the imaginary part of the Green's function vanishes below $2 m_{t}$. The qualitative difference between the solid and the short-dashed curves will be reflected in the comparison of our final results for the invariant-mass distribution with the prediction based on a fixed-order calculation: for the color-singlet curve we observe a sizable excess in the region below the nominal threshold up to roughly $5 \mathrm{GeV}$ above. In the color-octet case, as a consequence of the relative smallness of $C^{[8]}$, the prediction follows roughly the Born approximation. Although the color-octet Green's function is significantly smaller than the singlet one, the relatively large hard scattering factor $\mathcal{L} \otimes F$ for ${ }^{1} S_{0}^{[8]}$ plus ${ }^{3} S_{1}^{[8]}$, which exceeds the one for the singlet case by roughly a factor 4 , quickly over-compensates the effect of the Green's functions.

In the present paper we use the analytical result of the Green's function, which includes the $\alpha_{\mathrm{s}}$ correction (i.e. the second term in the square brackets of (5.1)) by means of the Rayleigh-Schrödinger perturbation approach. In [20] a numerical solution to (5.2) has been employed, which resums the $\alpha_{\mathrm{s}}$ corrections to all order. The numerical solution is more stable against scale variation and is applicable over a wide range of $\mu_{\mathrm{r}}$. However, the difference between the two approaches is below $2 \%$ and formally of higher order. Extensive studies on higher-order effects to the color-singlet Green's function exist in the literature (see, e.g., [8, 43]), including different implementations of the Green's function. From the experience collected in the linear collider studies on $t \bar{t}$ production, we expect rather large corrections from the variation of $\mu_{\mathrm{r}}$ for the color-singlet Green's function of about $20 \%$ which is significantly bigger than the estimate from the NNLO Green's function mentioned above. In contrast to the color-singlet case the higher-order corrections to the color-octet Green's function are expected to be unimportant, since there is no resonance enhancement and the color coefficient $C^{[8]}$ is small.

\section{Invariant-mass distribution}

We are now in the position to combine the results of the preceding sections and discuss the cross section for the invariant top-quark distribution.

In Fig. 6.1 the invariant-mass distributions for LHC $(\sqrt{S}=14 \mathrm{TeV})$ is shown for the three dominant processes. The bands reflect the scale variation of the convolution $\mathcal{L} \otimes F$, which for the color-singlet case amounts to roughly $\pm 1 \%$. The reduction as compared to Table 3.2 and Fig. 6.1 is due to a compensation of the $\mu$ dependence after including the sub-leading NLO processes. Note, however, that the corresponding Green's function shows an uncertainty due to the renormalization-scale variation of about $20 \%$ which is well known from top-quark production studies in $e^{+} e^{-}$ collisions, consistent with the difference between solid and dash-dotted curves in Fig. 5.1 and thus not discussed in Fig. 6.1. This pattern is also evident from Fig. 6.2, where all production channels as listed in Table 3.2 are included. The width of the bands is obtained from varying renormalization and factorization scales in the hard cross section as described above. The additional uncertainty from the Green's function, which we estimate $20 \%$ for the singlet and below $5 \%$ for the octet case, is not included.

As expected, for $M<2 m_{t}$ the production of $t \bar{t}$ pairs is dominated by the singlet contribution. However, for $M>$ $2 m_{t}$ one observes a strong increase of the octet contributions, in particular of the gluon-induced subprocess which for $M \gtrsim 2 m_{t}+5 \mathrm{GeV}$ becomes even larger than the corresponding singlet contribution. For the color-octet case the scale dependence of the hard scattering amounts to $\pm 7 \%$. Considering the threshold behavior as shown in Figs. 6.1

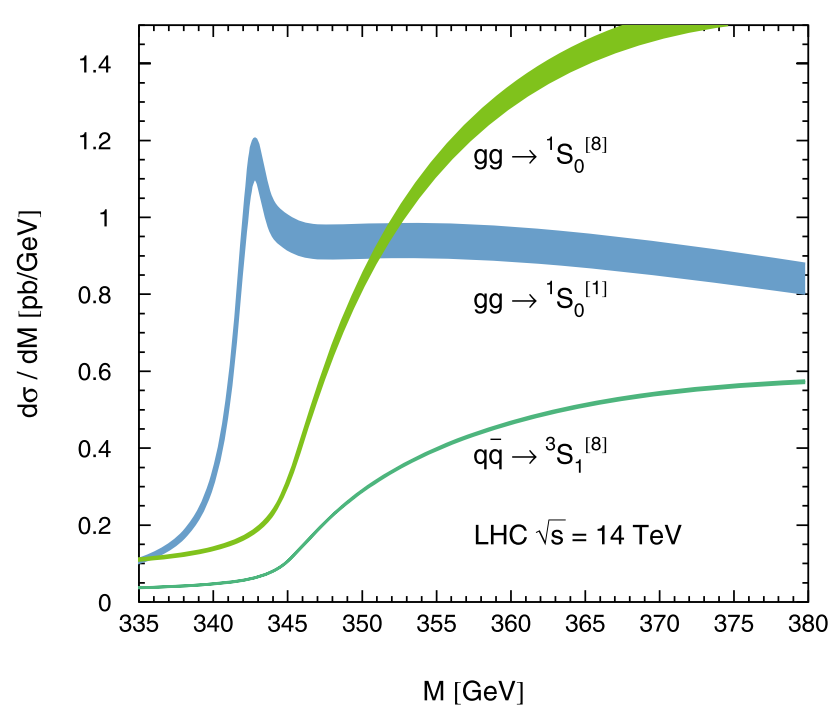

Fig. 6.1 (Color online) Invariant-mass distributions for leading subprocesses: $g g \rightarrow{ }^{1} S_{0}^{[1,8]}$ (blue and light green, respectively) and $q \bar{q} \rightarrow{ }^{3} S_{1}^{[8]}$ (green). For each process the bands take into account scale variation of the hard cross sections 
and 6.2 it is clear, that the location of the threshold is entirely governed by the behavior of the color-singlet ( $S$-wave) contribution. Thus, as a matter of principle, determining the location of this step experimentally would allow for a topquark mass measurement, which is conceptually very different from the one based on the reconstruction of a (colored) single quark in the decay chain $t \rightarrow W b$. In fact, much of the detailed investigations of $t \bar{t}$ threshold production at a linear collider were performed for this particular relations between the location of the color-singlet quasi-bound-state pole of $t \bar{t}$ and the top-quark $\overline{\mathrm{MS}}$-mass. The absolute normalization of the cross section is also sensitive to electroweak corrections [45-49], which are of the order of 5\% close to threshold. For example, the difference between corrections from a light $\left(M_{\mathrm{h}}=120 \mathrm{GeV}\right)$ and a heavy $\left(M_{\mathrm{h}}=1000 \mathrm{GeV}\right)$ Higgs boson amounts to roughly $6 \%$ [48].

In Fig. 6.3 the prediction for $\mathrm{d} \sigma / \mathrm{d} M$ based on NRQCD is compared with the one obtained from a fixed-order NLO calculation for stable top quarks which is obtained using the program HVQMNR [50]. As expected from the comparison of solid and dotted curves in Fig. 5.1, the two predictions overlap for invariant masses around $355 \mathrm{GeV}$. Above $355 \mathrm{GeV}$ relativistic corrections start to become important. From this comparison we find an additional contribution to the total cross section for $t \bar{t}$ production of roughly $10 \mathrm{pb}$, which could become of relevance for precision measurements. Note that the band of the NRQCD-based prediction only contains the uncertainty from the scale variation of $\mathcal{L} \otimes F$, whereas the one of the Green's function (which can reach up to $20 \%$; see Sect. 5) is not shown.

The analysis of this work has concentrated on the threshold region and is applicable for $M$ up $360 \mathrm{GeV}$ at most.

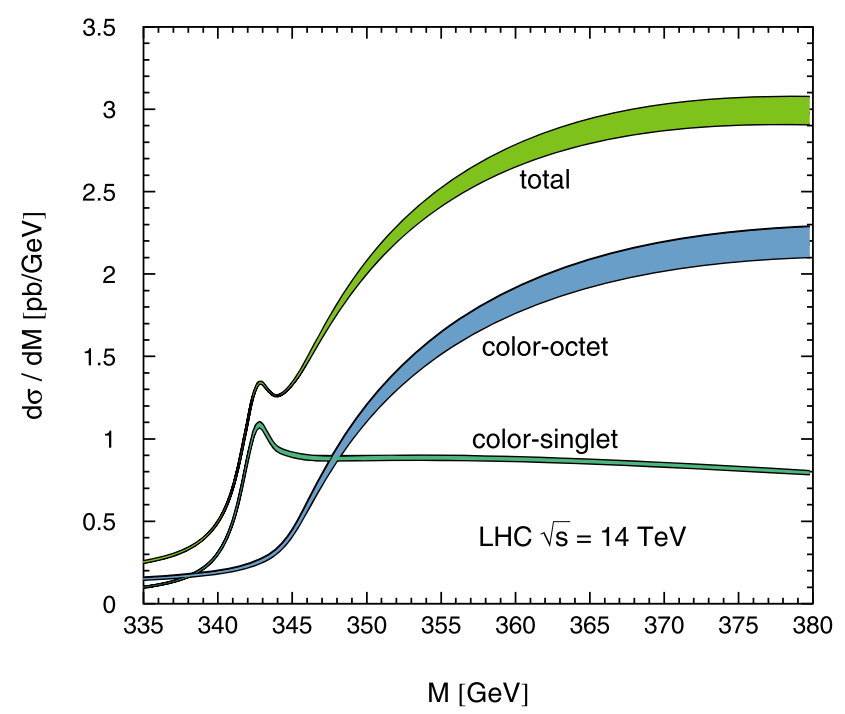

Fig. 6.2 Invariant-mass distribution including all production channels shown in Table 3.2. The width of the bands reflect the scale dependence of the hard scattering parts
However, it is obvious that the overall shape of $\mathrm{d} \sigma / \mathrm{d} M$ will be distorted and the mean $\langle M\rangle$ shifted to smaller values, which might affect the global fit of $\mathrm{d} \sigma / \mathrm{d} M$. In Fig. 6.4 we present for comparison the NLO prediction for $\mathrm{d} \sigma / \mathrm{d} M$ in the wide range up to $700 \mathrm{GeV}$. The distribution reaches quickly its maximum of $3.3 \mathrm{pb} / \mathrm{GeV}$ at around $390 \mathrm{GeV}$ and then falls off slowly. It is remarkable that its value at $370 \mathrm{GeV}$ is already not too far from the maximum of the curve, and the threshold modifications thus affect a sizeable part of the distribution.

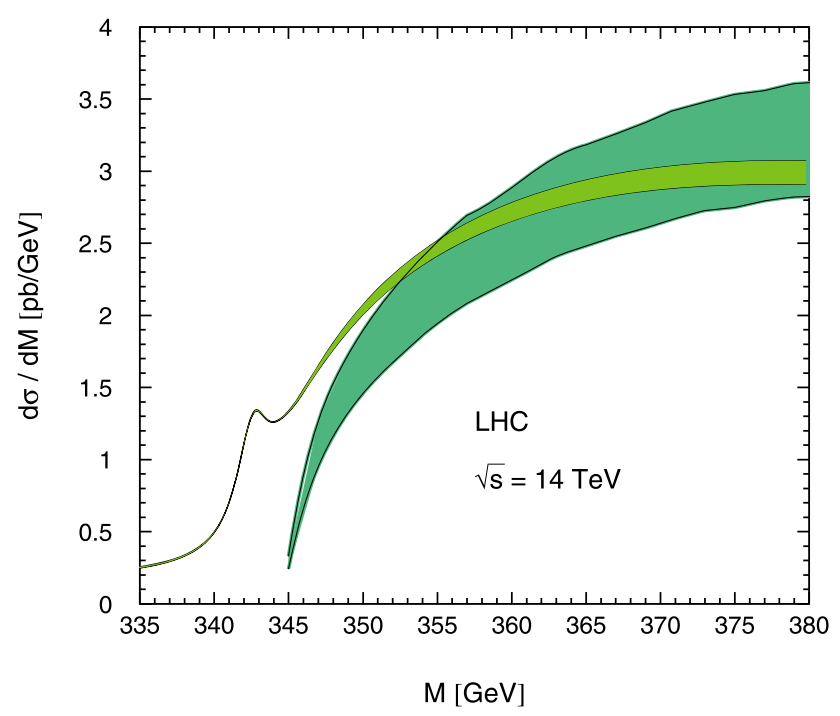

Fig. 6.3 Invariant-mass distribution $\mathrm{d} \sigma / \mathrm{d} M$ from NRQCD and for a fixed NLO for LHC with $\sqrt{s}=14 \mathrm{TeV}$. The bands are due to scale variation from $m_{t}$ to $4 m_{t}$. For the NRQCD prediction the additional uncertainty due to the Green's function estimated to $20 \%$ (5\%) for the color-singlet (-octet) contribution is not included

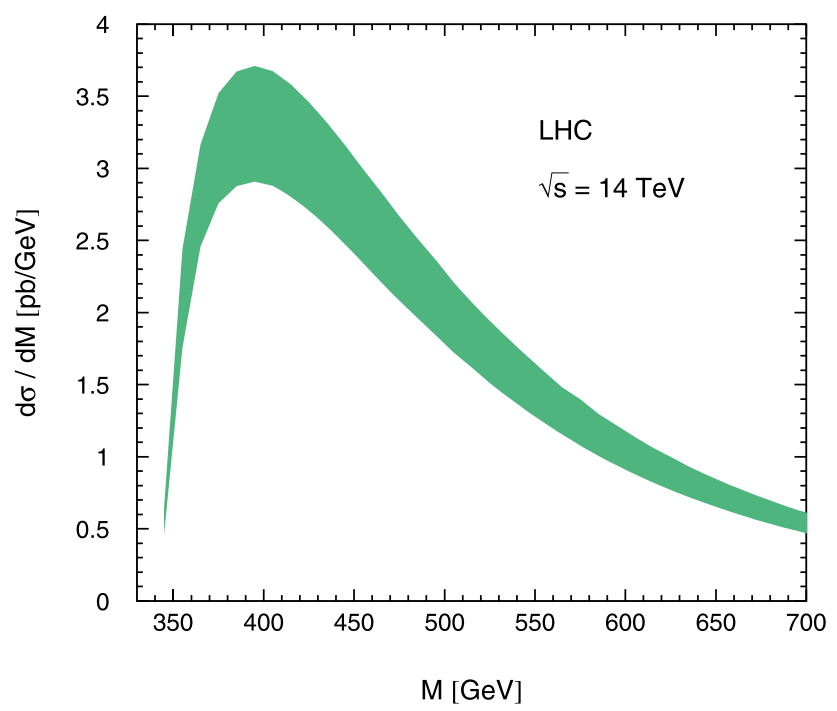

Fig. 6.4 Invariant-mass distribution $\mathrm{d} \sigma / \mathrm{d} M$ from NLO calculation for LHC with $\sqrt{s}=14 \mathrm{TeV}$ 
Although the most detailed top-quark studies will be performed at the LHC at an energy of $14 \mathrm{TeV}$, a sample of top quarks has been collected at the Tevatron in protonantiproton collisions at $1.96 \mathrm{TeV}$. Furthermore, the first LHC data set will be taken at $10 \mathrm{TeV}$. For this reason we give the results for these two cases; see Figs. 6.5 and 6.6. The cross section in Fig. 6.5 has the same characteristic shape as the one in Fig. 6.2; however, the absolute size is considerably smaller. As expected, the enhancement at threshold is significantly less pronounced for Tevatron where the colorsinglet contribution is very small.

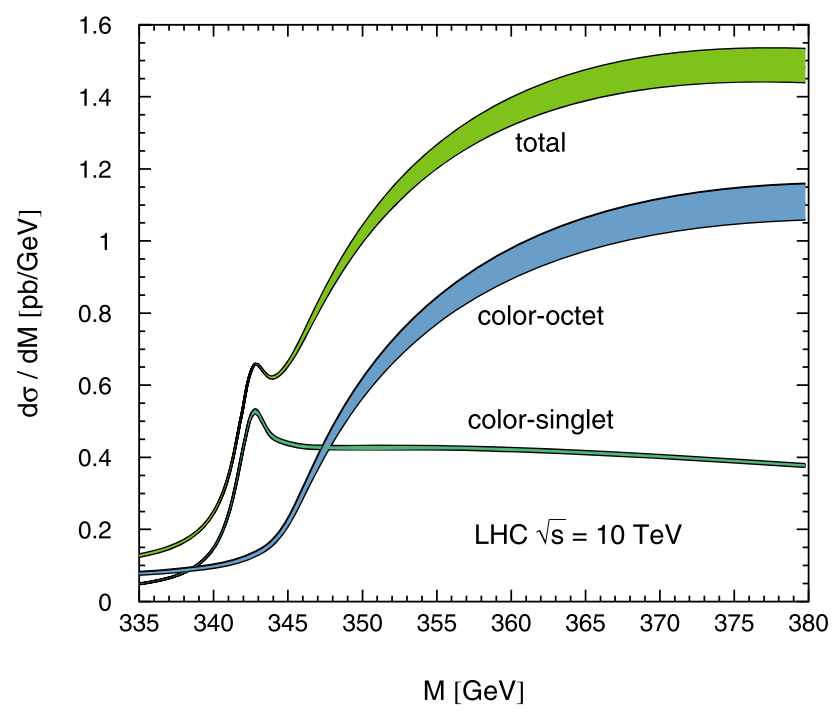

Fig. 6.5 Invariant-mass distribution $\mathrm{d} \sigma / \mathrm{d} M$ for LHC with $\sqrt{s}=10 \mathrm{TeV}$

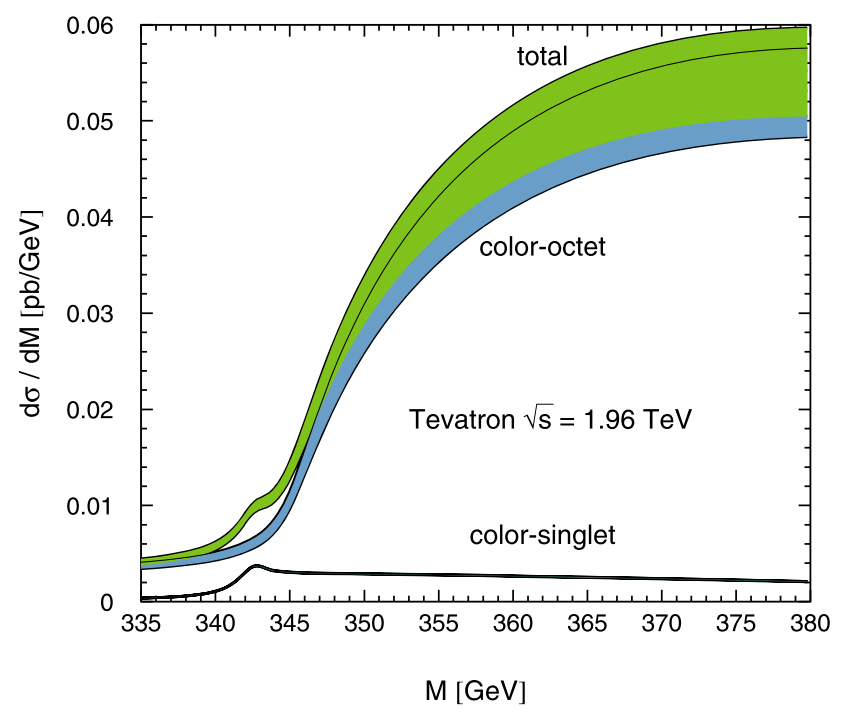

Fig. 6.6 Invariant-mass distribution $\mathrm{d} \sigma / \mathrm{d} M$ for Tevatron with $\sqrt{s}=1.96 \mathrm{TeV}$. At the Tevatron $q \bar{q} \rightarrow{ }^{1} S_{0}^{[8]}$ dominates the cross section, and the luminosity for the $g g$ channels is small; thus the bound-state peak is buried by color-octet production
Our analysis confirms the findings of [20]; however, the numerical results for the cross sections as presented in Fig. 6.2 are slightly higher than the corresponding corrections of [20], which is due to the combined effect of the softgluon resummation, the inclusion of the NLO sub-processes and the different matching to full QCD.

\section{Summary}

A NLO analysis of top-quark production near threshold at hadron colliders has been performed. The large width of the top quark in combination with the large contribution from gluon fusion into a (loosely bound) color-singlet $t \bar{t}$ system leads to a sizable cross section for masses of the $t \bar{t}$ system significantly below the nominal threshold. A precise measurement of the $M_{t \bar{t}}$-distribution in this region, which is dominated by the color-singlet configuration, could lead to a top-quark mass determination which does not involve the systematic uncertainties inherent in the determination of the mass of a single (color triplet) quark. Furthermore, also the shape of the differential distribution $\mathrm{d} \sigma / \mathrm{d} M$ is distorted and the mean $\langle M\rangle$ shifted towards smaller values.

The effects of initial-state radiation as well as bound-state corrections are taken into account in a consistent manner at NLO. As compared to [20] we include the complete $\hat{s}$ dependence in the matching condition and also implement all NLO sub-processes. We observe a partial numerical cancellation between these two effects leading to similar predictions as [20]. Furthermore, we perform a soft-gluon resummation and thus include the dominant logarithmically enhanced higher-order terms. This last step stabilizes the prediction. However, it enhances the cross section at most by $10 \%$.

The effects are more pronounced at the LHC with top production being dominated by gluon fusion and less relevant in proton-antiproton collisions with top quarks dominantly in color-octet states. Considering the threshold region (say up to $M_{t \bar{t}}=350 \mathrm{GeV}$ ) separately, an integrated cross section of $15 \mathrm{pb}$ is obtained, which should be compared to $5 \mathrm{pb}$ as derived from the NLO predictions using a stable top quark and neglecting the binding correction. Within this relatively narrow region, the enhancement amounts to roughly a factor 3 and a significant shift of the threshold. Compared to the total cross section for $t \bar{t}$ production of about $840 \mathrm{pb}$ (obtained using fixed-order NLO accuracy for $\mu=m_{t}$; see, e.g., [25]), the increase is relatively small, about $1 \%$. However, in view of the anticipated experimental precision of better than $10 \%$, these effects should not be ignored.

Note added

While this article was finished an analytic evaluation of the total cross section at NLO accuracy appeared [51], which 
has been used in [20] to clarify the existence of a nondecoupling top-quark effect overlooked in [19] (see [20, footnote 3, p. 73].

Acknowledgements This work was supported by the DFG through SFB/TR 9, by the BMBF through contract 05HT4VKAI3 and by the Helmholtz Gemeinschaft under contract VH-NG-105.

\section{References}

1. E. Vanes, Plenary Talk at ICHEP 08, Philadelphia, USA, Jul. 29thAug. 5th, 2008

2. ATLAS-TDR-015 (1999)

3. CMS-TDR-008-2, 2006, http://cmsdoc.cern.ch/cms/cpt/tdr/

4. G.T. Bodwin, E. Braaten, G.P. Lepage, Phys. Rev. D 51, 1125 (1995). hep-ph/9407339

5. G.T. Bodwin, E. Braaten, G.P. Lepage, Phys. Rev. D 55, 5853 (1997)

6. M. Martinez, R. Miquel, Eur. Phys. J. C 27, 49 (2003). hep-ph/0207315

7. K. Fujii, T. Matsui, Y. Sumino, Phys. Rev. D 50, 4341 (1994)

8. A.H. Hoang et al., Eur. Phys. J. C 2, 1 (2000). hep-ph/0001286

9. V.S. Fadin, V.A. Khoze, JETP Lett. 46, 525 (1987)

10. M.J. Strassler, M.E. Peskin, Phys. Rev. D 43, 1500 (1991)

11. M. Jezabek, J.H. Kuhn, T. Teubner, Z. Phys. C 56, 653 (1992)

12. Y. Sumino, K. Fujii, K. Hagiwara, H. Murayama, C.K. Ng, Phys. Rev. D 47, 56 (1993)

13. A.H. Hoang, Acta Phys. Polon. B 34, 4491 (2003). hep-ph/ 0310301

14. A. Pineda, A. Signer, Nucl. Phys. B 762, 67 (2007). hep-ph/ 0607239

15. M. Beneke, Y. Kiyo, K. Schuller, Phys. Lett. B 658, 222 (2008). 0705.4518

16. M. Beneke, Y. Kiyo, A.A. Penin, Phys. Lett. B 653, 53 (2007). 0706.2733

17. M. Beneke, Y. Kiyo, Phys. Lett. B 668, 143 (2008). 0804.4004

18. J.H. Kuhn, E. Mirkes, Phys. Rev. D 48, 179 (1993). hep-ph/ 9301204

19. A. Petrelli, M. Cacciari, M. Greco, F. Maltoni, M.L. Mangano, Nucl. Phys. B 514, 245 (1998). hep-ph/9707223

20. K. Hagiwara, Y. Sumino, H. Yokoya, Phys. Lett. B 666, 71 (2008). 0804.1014

21. V.S. Fadin, V.A. Khoze, T. Sjostrand, Z. Phys. C 48, 613 (1990)

22. P. Nason, S. Dawson, R.K. Ellis, Nucl. Phys. B 303, 607 (1988)

23. W. Beenakker, H. Kuijf, W.L. van Neerven, J. Smith, Phys. Rev. D 40, 54 (1989)
24. W. Bernreuther, A. Brandenburg, Z.G. Si, P. Uwer, Nucl. Phys. B 690, 81 (2004). hep-ph/0403035

25. S. Moch, P. Uwer, Phys. Rev. D 78, 034003 (2008). 0804.1476

26. S. Moch, P. Uwer, Nucl. Phys. Proc. Suppl. 183, 75 (2008). 0807.2794

27. M. Cacciari, S. Frixione, M.L. Mangano, P. Nason, G. Ridolfi, J. High Energy Phys. 09, 127 (2008). 0804.2800

28. N. Kidonakis, R. Vogt, Phys. Rev. D 78, 074005 (2008). 0805.3844

29. R. Frederix, F. Maltoni, 0712.2355 (2007)

30. W.K. Tung et al., J. High Energy Phys. 02, 053 (2007). hep-ph/ 0611254

31. K.G. Chetyrkin, J.H. Kuhn, M. Steinhauser, Comput. Phys. Commun. 133, 43 (2000). hep-ph/0004189

32. M. Cacciari, Nucl. Phys. B 571, 185 (2000). hep-ph/9910412

33. S. Moch, J.A.M. Vermaseren, A. Vogt, Nucl. Phys. B 726, 317 (2005). hep-ph/0506288

34. H. Contopanagos, E. Laenen, G. Sterman, Nucl. Phys. B 484, 303 (1997). hep-ph/9604313

35. S. Catani, M.L. Mangano, P. Nason, L. Trentadue, Nucl. Phys. B 478, 273 (1996). hep-ph/9604351

36. J. Kodaira, L. Trentadue, Phys. Lett. B 112, 66 (1982)

37. R. Bonciani, S. Catani, M.L. Mangano, P. Nason, Nucl. Phys. B 529, 424 (1998). hep-ph/9801375

38. R. Bonciani, S. Catani, M.L. Mangano, P. Nason, Nucl. Phys. B 803, 234 (2008)

39. M. Beneke, A. Signer, V.A. Smirnov, Phys. Lett. B 454, 137 (1999). hep-ph/9903260

40. M. Jezabek, J.H. Kuhn, Phys. Rev. D 48, 1910 (1993). hep-ph/ 9302295

41. A. Czarnecki, K. Melnikov, Nucl. Phys. B 544, 520 (1999). hep-ph/9806244

42. K.G. Chetyrkin, R. Harlander, T. Seidensticker, M. Steinhauser, Phys. Rev. D 60, 114015 (1999). hep-ph/9906273

43. M. Beneke, Y. Kiyo, K. Schuller, Nucl. Phys. B 714, 67 (2005). hep-ph/0501289

44. M. Beneke, Phys. Lett. B 434, 115 (1998). hep-ph/9804241

45. W. Beenakker et al., Nucl. Phys. B 411, 343 (1994)

46. J.H. Kuhn, A. Scharf, P. Uwer, Eur. Phys. J. C 45, 139 (2006). hep-ph/0508092

47. W. Bernreuther, M. Fucker, Z.G. Si, Int. J. Mod. Phys. A 21, 914 (2006). hep-ph/0509210

48. J.H. Kuhn, A. Scharf, P. Uwer, Eur. Phys. J. C 51, 37 (2007). hep-ph/0610335

49. W. Bernreuther, M. Fucker, Z.-G. Si, Phys. Rev. D 74, 113005 (2006). hep-ph/0610334

50. M.L. Mangano, P. Nason, G. Ridolfi, Nucl. Phys. B 373, 295 (1992)

51. M. Czakon, A. Mitov, 0811.4119 (2008) 